\title{
La tradition des autobiographies chantées chez les Sharanahua (Amazonie occidentale)
}

La tradición de cantos autobiográficos entre los sharanahua (Amazonía occidental)

Traditional Autobiographical Songs among the Sharanahua (Western Amazon)

\section{Pierre Déléage}

\section{(2) OpenEdition}

Journals

\section{Édition électronique}

URL : http://journals.openedition.org/bifea/3011

DOI : 10.4000/bifea.3011

ISSN : 2076-5827

\section{Éditeur}

Institut Français d'Études Andines

\section{Édition imprimée}

Date de publication : 1 décembre 2008

Pagination : 535-551

ISSN : 0303-7495

\section{Référence électronique}

Pierre Déléage, « La tradition des autobiographies chantées chez les Sharanahua (Amazonie occidentale) », Bulletin de l'Institut français d'études andines [En ligne], 37 (3) | 2008, mis en ligne le 01 juin 2009, consulté le 27 novembre 2020. URL : http://journals.openedition.org/bifea/3011 ; DOI : https://doi.org/10.4000/bifea.3011

\section{(ब) $\odot \Theta$}

Les contenus du Bulletin de l'Institut français d'études andines sont mis à disposition selon les termes de la licence Creative Commons Attribution - Pas d'Utilisation Commerciale - Pas de Modification 4.0 International. 


\title{
La tradition des autobiographies chantées chez les Sharanahua (Amazonie occidentale)
}

\author{
Pierre Déléage*
}

\begin{abstract}
Résumé
Les Sharanahua (peuple pano d'Amazonie péruvienne) sont dépositaires d'une tradition de chants autobiographiques. Deux transcriptions et traductions de ceux-ci sont ici proposées. Une analyse détaillée fait apparaître deux aspects problématiques. Ces chants narrent des souvenirs très personnels dans un langage pourtant extrêmement stéréotypé et ils sont présentés à la fois comme des citations des paroles passées du chanteur et comme la répétition fidèle de paroles qui firent l'objet d'un enseignement codifié. Cet article explore les implications de ces apparents paradoxes.
\end{abstract}

Mots clés : chants traditionnels, autobiographie, pragmatique, transmission, Sharanahua (Amazonie)

\section{La tradición de cantos autobiográficos entre los sharanahua (Amazonía occidental)}

\section{Resumen}

Los sharanahua, que conforman un grupo pano de la Amazonía occidental peruana, mantienen una tradición de cantos autobiográphicos. Dos transcriptiones y traducciones de estos cantos están brindadas en este artículo. Su análisis detallado hace resaltar dos puntos problemáticos. Por una parte, estos cantos alunden a recuerdos muy personales, pero utilizando un lenguaje extremadamente estereotipado. Por otra parte, son presentados como repeticiones de los discursos pasados del cantor y a la par repeticiones exactas de palabras enseñadas. Este artículo explora las implicaciones de estas aparentes paradojas.

Palabras clave: cantos tradicionales, autobiografía, pragmática, transmission, sharanahua (Amazonía)

Laboratoire d'anthropologie sociale, 52 rue Cardinal Lemoine, 75005 Paris - France. Department of Anthropology, UC Berkeley, 232 Kroeber Hall, Berkeley CA 94720 - USA. E-mail: deleagepierre@hotmail.com 


\title{
Traditional Autobiographical Songs among the Sharanahua (Western Amazon)
}

\begin{abstract}
The Sharanahua (a Panoan people of Western Amazon) maintain a tradition of autobiographical songs. Two transcriptions and translations of such songs are presented here. A detailed analysis highlights two main problems. These songs narrate very personal memories, but are couched in an extremely stereotypical language; secondly, they are presented as simultaneous quotations of past speech of the singer and as faithful repetitions of taught words. This text explores the implications of these apparent paradoxes.
\end{abstract}

Key words: traditional songs, autobiography, pragmatics, transmission, Sharanahua (Amazon)

Percy 1 était un Sharanahua du village de Gasta Bala. Il avait depuis quelques années installé sa maison un peu l'écart de la communauté, sur la rive même du fleuve Purus. Il y vivait avec son épouse et deux de ses filles. Ses frères étaient partis, depuis longtemps déjà, rendre visite aux Yaminahua et Yahuanahua du Brésil. Ils avaient rencontré leur femme làbas, au sein de ces sociétés linguistiquement et culturellement très proches, et ils y étaient restés, dans des villages situés à proximité de nombreuses implantations de métis brésiliens. Souvent, Percy disait combien cette partie de sa famille lui manquait ; une vingtaine d'années auparavant, il était allé les voir, une seule fois mais pendant une année entière, et il en conservait un souvenir ému.

Il avait, quant à lui, choisi de revenir sur les rives péruviennes du fleuve Purus. Il s'y était marié très tôt, avec la fille sharanahua d'un homme important. De leur union étaient nés plusieurs enfants. Cependant, Percy n'était pas réputé pour sa fidélité : dès son premier enfant, il avait connu de nombreuses autres femmes. II avait même pensé, à plusieurs reprises, officialiser au moins une de ces relations extraconjugales. Mais sa femme s'y était toujours énergiquement opposée, appuyée par son père. Progressivement, il s'était fait à cette idée et il avait abandonné ses amours extraconjugaux. La réconciliation avec sa femme, et surtout avec sa belle famille, avait pris la forme d'un processus lent et difficile, parsemé de moments douloureux sur lesquels ils n'aimait pas trop s'attarder.

Lorsque nous l'avons rencontré pour la première fois, en 2001, il avait déjà atteint un âge avancé. On disait maintenant de lui qu'il était trop vieux pour séduire de nouvelles femmes. Son foyer s'était donc stabilisé et c'est avec une pointe de nostalgie qu'il se souvenait de temps en temps de ses conquêtes passées. Mais Percy était également un bon chanteur : il disposait d'un répertoire de chants traditionnels qui lui permettaient d'évoquer, à voix haute, ces différents souvenirs. Ces chants, contrairement à ceux que nous commencions à bien connaître, n'avaient aucun rapport avec le chamanisme. Ils étaient, si l'on veut, « profanes», ne faisant intervenir aucune entité surnaturelle, ni dans le contenu de leurs énoncés, ni dans les conditions de leur énonciation. Ils narraient, dans un langage sibyllin, certains épisodes de la vie de leur énonciateur, tous empreints d'une forte charge émotionnelle - ces épisodes que nous venons de retracer brièvement. En un mot, ces chants appartenaient à la tradition des récits autobiographiques des Sharanahua².

1 Il s'agit là d'un pseudonyme.

2 Les Sharanahua parlent une langue pano et comptent près de 650 membres. Ils vivent au sein de villages d'environ 150 habitants chacun, dispersés sur les rives péruviennes du fleuve Purus, au cœur du bassin amazonien. Ils ont pour 


\section{UNE TRADITION AUTOBIOGRAPHIQUE}

Les Sharanahua considèrent les chants *yama yama comme un genre particulier de discours. Ils leur permettent d'exprimer, dans un langage standardisé, le souvenir d'évènements passés. II s'agit, à vrai dire, toujours de souvenirs personnels énoncés du point de vue subjectif du chanteur. C'est dans cette mesure qu'il est légitime de les qualifier d'autobiographiques. Cependant, il ne faut en aucun cas les confondre avec les discours ordinaires qui prennent parfois une allure autobiographique, lorsqu'il est question de se remémorer quelque anecdote issue d'un passé commun ou d'édifier un jeune auditoire en conférant un caractère exemplaire au récit d'expériences personnelles judicieusement choisies. Dans ces cas, il est effectivement possible de parler d'autobiographies, mais elles diffèrent alors profondément des chants *yama yama que l'on peut qualifier, par contraste avec ces discours ordinaires, de discours traditionnels.

Comment les Sharanahua définissent-ils ce genre traditionnel de discours autobiographique? Ils le nomment fana, « chant». Quelquefois, pour les besoins immédiats de la conversation avec l'ethnologue, on l'appelle *yama yama, en reprenant son refrain distinctif, mais cette terminologie est loin d'être acceptée par tous. Ce refrain est d'ailleurs, selon les Sharanahua, dénué de tout sens : il ne sert qu'à rythmer l'énonciation, à séparer les lignes les unes des autres3. Le terme n'évoque certes pas les seuls chants autobiographiques : tous les chants, initiatiques, chamaniques, autobiographiques ou même étrangers, peuvent être nommés fana. Toutefois une telle caractérisation permet de les distinguer nettement du discours ordinaire en mettant en avant une structure mélodique, une métrique assez régulière et une indéniable tendance à la répétition. À ces critères qui définissent les chants en général, on peut ajouter, en ce qui concerne les Sharanahua, l'utilisation d'un langage relativement obscur, voire, comme nous venons de le noter, de termes dénués de signification. L'ensemble de ces éléments concourent à la constitution d'un genre discursif particulier qui doit faire l'objet d'un apprentissage bien défini contrairement aux discours quotidiens que l'on apprend à prononcer sans enseignement reconnu comme tel.

De ce point de vue, un aspect de ces chants doit d'ores et déjà être souligné : leur compréhension n'est pas immédiate. Comprendre un chant *yama yama, exige le décodage d'un vocabulaire spécifique composé pour l'essentiel de termes ésotériques qui se retrouvent d'un chant à l'autre. Ces termes ne sont ni des emprunts à des langues étrangères, ni des archaïsmes, ni des créations idiosyncrasiques ; on peut les qualifier de « substituts » dans la mesure où ils viennent remplacer, de manière systématique, certains termes précis de la langue quotidienne. Leur emploi répété permet à n'importe quel auditeur, non pas de comprendre le chant, mais de le reconnaître en tant qu'appartenant au genre des *yama

voisins d'autres groupes pano, tels que les Mastanahua, les Amahuaca ou les Cashinahua, mais aussi les Madihá (Culina), de langue arawa, ou les Yine (Piro), de langue arawak. Les informations présentées ici proviennent d'un seul village du Purus, dénommé Gasta Bala, où nous avons séjourné pendant 19 mois, échelonnés d'octobre 2001 à août 2004. Les Sharanahua, originaires des affluents du fleuve Jurua, sont arrivés dans la région vers le milieu du siècle dernier. Ils vivent aujourd'hui, pour l'essentiel, de l'horticulture du manioc et de la banane plantain, associée à des activités de chasse et de pêche, hautement valorisées. Leurs relations sociales restent régies par un système dualiste formé de deux moitiés exogamiques : les roa adifo (« les anciens parfaits ») et les nahua facufo (« les enfants des étrangers »).

3 Une interprétation étymologique, qui n'engage que la responsabilité de l'auteur, pourrait prendre comme point de départ le terme yaba dans la mesure où $/ \mathrm{m} /$ et $/ \mathrm{b} /$ sont allophones en sharanahua. Selon les contextes, yaba peut signifier «mort » (substantif) ou « ne pas exister» (verboïde). Par exemple, pour exprimer le fait qu'il n’y a personne quelque part, on peut dire : yora yabai où yora signifie « quelqu'un » et où /yaba-i/ apparaît comme un verbe conjugué à l'aspect progressif. Si on accepte une telle interprétation, le refrain ne serait qu'une manière redoublée d'exprimer une « absence » : celle des évènements passés évoqués dans le chant. 
yama. De ce fait, ces chants autobiographiques ressemblent aux chants chamaniques des Sharanahua qui présentent la même forme d'opacité ; il faut néanmoins remarquer que dans ce dernier cas, l'opacité résulte d'un principe assez clair : c'est une manière de donner à entendre à l'auditeur non initié que le chant est énoncé en un langage non humain. Les chants *yama yama, quant à eux, ne sont chantés dans un langage crypté pour aucune raison essentielle, au dire des Sharanahua. Cette absence de justification constituera le premier problème de cet article.

Les chants *yama yama se définissent donc aussi bien par leur forme que par leur contenu. Les Sharanahua affirment aujourd'hui que ce genre discursif n'a d'autre finalité que l'expression des émotions du chanteur. Lorsque quelqu'un se remémore, de manière précise et insistante, des évènements de sa vie passée dotés d'une forte charge émotionnelle, il chante un *yama yama. Cela ne lui permet pas de chasser sa tristesse ou sa nostalgie, encore moins, dit-on, de les communiquer à d'autres — c'est là simplement ce qu'il est possible de faire et ce que certains savent faire et font.

Pour désigner le type d'émotion située en corrélation avec l'énonciation des chants autobiographiques, les Sharanahua utilisent le verbe /shina-/ qui peut être traduit par « penser », « imaginer » et surtout « se souvenir ». Il est alors employé dans des constructions telles que /ichapa shina-/ ou /fasi shina-/ introduisant l'idée d'excès : on « pense trop », on «se souvient trop »4. L'expression traduit simplement un état psychologique où le sujet est affecté par la saillance démesurée de ses souvenirs et des émotions qui leur correspondent. Il s'agit toujours là de tristesse : celle liée au regret, à la nostalgie de joies passées ou au souvenir encore vivant de peines révolues.

Toutefois, le registre des évènements passés évoqués par les chants *yama yama est très limité. On en compte deux : les voyages et les amours. Et à vrai dire, les chants décrivant des voyages sont aussi souvent des chants d'amour. Dans tous les cas, au centre du chant se situent une ou plusieurs personnes que le locuteur a aimées.

Il faut néanmoins préciser que les anciens racontent que, fut un temps, ces chants jouèrent un rôle important au cours de la séduction d'un être aimé. Ils permettaient, grâce à leur langage cryptique, d'exprimer les sentiments que l'on éprouvait vis-à-vis d'une personne sans avoir à les lui communiquer directement, dans la crudité du langage quotidien. Les chants exposaient alors les qualités de l'être aimé et celles du chanteur. Aujourd'hui, la séduction se passe de ce moyen de communication indirect; les jeunes ne voient plus guère la nécessité d'apprendre ce langage compliqué, saturé de termes dont le sens et la motivation ont maintenant largement disparu. II nous semble toutefois que le simple fait que les chanteurs insistent beaucoup sur cette ancienne fonction (réelle ou supposée) des *yama yama fournit un indice pertinent quant au statut qu'il faut leur conférer.

Il est en effet difficile d'accepter les affirmations rapides des Sharanahua lorsqu'ils définissent les *yama yama comme de simples « expressions » d'émotions. Il faut d'abord noter que nous n'avons jamais observé quelqu'un entonnant ses chants seul, comme il est de coutume de le faire, par exemple, pour les lamentations funéraires. II va presque de soi qu'un auditoire est nécessaire à leur énonciation, même si cela n'est jamais spécifié comme tel. De plus, l'utilisation du langage, même avec une simple intention expressive, revient toujours à communiquer quelque chose à autrui. Par ailleurs, on comprendrait mal I'utilité de l'apprentissage d'un genre discursif relativement complexe et encore moins la réussite de sa transmission, s'il n'y avait pas là en jeu quelque chose de plus fondamental.

4 /ichapa/ signifie « beaucoup » et /fasi/ «trop ». La construction /shina-pai-/ dénote un acte de remémoration volontaire ; /shina-funo-/, /penser-perdre/, signifie « oublier»; la « tristesse » est nommée shinamitsa. Mary Scott traduit le verbe /shina-/ par « se lamenter», « penser» et « se rappeler» (Scott, $2004: 62)$. 
Après tout, il est possible d'exprimer ses émotions de multiples manières et on ne voit guère pourquoi serait transmise une technique aussi coûteuse s'il n'y avait pas une bonne raison à cela. Il s'agira là du second problème de cet article.

Notre analyse commencera par la présentation de deux chants *yama yama, l'un décrivant un voyage, l'autre un épisode de la vie amoureuse du chanteur. Les deux chants ont été enregistrés durant la même après-midi, au cours d'une conversation à bâtons rompus où il était question de certains aspects de la vie du chanteur. Nous n'avons pas demandé expressément à les enregistrer : c'est Percy qui nous conseilla de le faire car ils étaient « plus beaux » que les chants chamaniques incompréhensibles qui nous obsédaient tellement. À ce moment-là, notre maîtrise de la langue sharanahua était encore assez rudimentaire, même si nous étions capable de reconnaître une bonne partie du vocabulaire des chants. Percy éclairait ensuite, dans un mélange d'espagnol et de sharanahua, la signification, propre au genre *yama yama, de certains mots, mais il resta vague quant au sens général des chants.

C'est plus tard, en compagnie de Jaime Del Aguila, puis d'Oswaldo Mélendez, que nous menâmes à bien la transcription puis la traduction de ces deux chants. Comme souvent, la première traduction fut désastreuse et nous dûmes faire de nouveau appel à Percy pour comprendre certains termes et certaines constructions ; il nous aida alors grandement tout en laissant plusieurs aspects du second chant dans l'ombre. C'est finalement Josefa Mélendez, elle-même chanteuse de *yama yama, qui nous livra les ultimes clefs interprétatives — nous verrons lesquelles.

\section{PREMIER CHANT, PAR PERCY, DE LA MOITIÉ ROA ADIFO}

1 yama yama

« un da udu baiqui

udu baiquiriri

ati cairocomun

5 yaba nahuacaifo

yaba nahuacaifo »

mun huahuadicodi

yama yama

yama yama

10 « ocu tsoayashomu

ocu tsoayashomu

da yaba nahuafo

oidicopaino

oidicopaino »

15 huahuadicohuurana

huahuadicohuurana

ati shomuncai

ati shomuncai

« da yaba nahuanu

20 ua pota namudi yama yama

« en aval de ce fleuve

en aval du fleuve

si nombreux

les étrangers morts

les étrangers morts »

ainsi ai-je dit

yama yama

yama yama

« au loin, avec qui?

au loin, avec qui ?

ces étrangers morts

je voudrais les voir

je voudrais les voir »

ainsi ai-je dit en y allant

ainsi ai-je dit en y allant

ils sont si loin

ils sont si loin

« ces étrangers morts

ils m'ont rejeté 
ua pota namudi »

idicocurana acuranara yama

yama yama

uhuun aco ishta

25 uhuun aco ishta

dicahuu ua chaca

dicahuu ua chaca

ocu yaba nahuacai

uhuun aco ayashon

30 mun tanadicodi

mun tanadicodi

yama yama

« ocu tsoayashomu

da yaba nahuafo

35 oidicopaino

oidicopaino »

da ua chacashta

da ua chacashta

huahuadicohuurana

huuranara yama

40 ua ati shomucai

uhuun aco ishta

dicahua amuti

dicahua amuti

ari ua chacashta

45 uhuun aco ayashon

uhuun aco ayashon

ocu yaba nahuacai

mun tanadicodi

mun tanadicodi

50 yama yama

ahuuscashtaquiroco

ahuuscashtaquiroco

uhuun aco ishta

dicahua amuti

55 huahuadicohuurana

huuranara yama

« ano noco rahuumai

ano noco rahuumai ils $\mathrm{m}^{\prime}$ ont rejeté »

ainsi ai-je dit en repartant yama

yama yama

« mon doux ishpingo

mon doux ishpingo

écoute ma tristesse

écoute ma tristesse

les lointains étrangers morts

mon ishpingo

je suis devenu [comme eux]

je suis devenu [comme eux]»

yama yama

« au loin, avec qui ?

ces étrangers morts

je voudrais les voir

je voudrais les voir»

moi, triste

moi, triste

ainsi ai-je dit, en y allant yama

« ils sont si loin de moi

mon doux ishpingo

écoute-moi

écoute-moi

moi, triste

mon ishpingo

mon ishpingo

les lointains étrangers morts

je suis devenu [comme eux]

je suis devenu [comme eux]»

yama yama

«pourquoi hélas?

pourquoi hélas?

mon doux ishpingo

écoute-moi »

ainsi ai-je dit, en y allant yama

« là, nous deux ensemble

là, nous deux ensemble 
La tradition des autobiographies chantées chez les Sharanahua

$\begin{array}{ll}\begin{array}{l}\text { uhuun aco ishtaya } \\ \text { didihuapaino » }\end{array} & \text { mon doux ishpingo } \\ \text { huahuadicohuurana } & \text { nous pourrions vivre» } \\ \text { huahuadicohuurana } & \text { ainsi ai-je dit, en y allant } \\ \text { yama yama } & \text { yama yama } \\ \text { ahuuscashtaquiroco } & \text { "pourquoi hélas? } \\ \text { «da yaba nahuanu } & \text { ces étrangers morts } \\ \begin{array}{l}\text { ati shomuncai } \\ \text { ua pota namudi » }\end{array} & \text { ils sont si loin } \\ \text { idicocurana curanara yama } & \text { ils m'ont rejeté » } \\ \text { yama yama } & \text { ainsi ai-je dit, en repartant yama } \\ & \text { yama yama } \\ \text { da udu baiqui } & \\ \text { udu uhua baiqui } & \text { "en aval du fleuve } \\ \text { ari ua chacafin } & \text { en aval du grand fleuve } \\ \text { ocu yaba nahuacai } & \text { moi, triste } \\ \text { tanadicoyotada } & \text { les lointains étrangers morts } \\ \text { yama yama } & \text { je suis devenu [comme eux]» } \\ \text { « ocu tsoayashomu } & \text { yama yama } \\ \text { yaba nahuacaifo } & \text { « au loin, avec qui ? } \\ \text { oidicopaino painora yama » } & \text { je voudrais les voir yama » } \\ \text { huahuadicohuurana } & \text { ainsi ai-je dit, en y allant } \\ \text { huahuadicohuurana } & \text { ainsi ai-je dit, en y allant }\end{array}$

\section{APERÇU GLOBAL ET DÉCRYPTAGE}

Nous ne souhaitons, pour le moment, que rendre intelligible le chant qui vient d'être lu. Le lecteur aura immédiatement compris qu'il s'agissait là d'un langage très particulier qui ne peut être compris sans commentaires extérieurs. Il faut d'abord reconnaître que le chant est composé de deux types d'énoncés : les premiers portent sur les conditions d'énonciation tandis que les seconds constituent l'objet du chant à proprement parler, l'énoncé des souvenirs du chanteur.

Ainsi, on remarque l'omniprésence de l'énoncé « Ainsi ai-je dit » (7, 15-16, 22, 39, 55, 60$61,67,78-79)$ qui définit le statut énonciatif du chant. La quasi-intégralité du chant peut donc être considérée comme du discours rapporté : le chanteur cite ses propres paroles passées. De ce point de vue, le chant n'apparaît que comme une simple répétition.

Dès lors, le chant ne décrit pas la situation actuelle d'énonciation, mais rappelle, très brièvement, les deux éléments fondamentaux qui caractérisèrent la situation passée d'énonciation des paroles citées. D'une part, le locuteur était alors « triste » (37-38), et d'autre part il prononça ces paroles au cours d'un voyage : « en y allant » $(15-16,39,55$, $60-61,78-79)$ et « en repartant» $(22,66)$. Ce sont ces deux aspects de l'acte de parole passé qui justifient sa reprise dans un chant *yama yama : une forte charge émotionnelle et le contexte d'un voyage. 
Le reste du chant doit donc être envisagé comme une forme d'auto-citation. Ces paroles furent adressées au frère du chanteur qui est explicitement placé en position d'auditeur : «Écoute-moi » (42-43, 54), «Écoute ma tristesse » (26-27). Cependant, son nom propre ne sera jamais prononcé : le terme qui s'y substitue est « ishpingo » (aco, 24-25, 29, 41, 45-46, 53, 58), un arbre au bois très dur (Amburana Cearensis).

Il s'agit là d'un des grands principes des chants *yama yama : à chaque nom propre se substitue un terme " appartenant » à une des deux moitiés exogamiques des Sharanahua : les roa adifo, les « anciens parfaits», ou les nahua facufo, les " enfants des étrangers». «Ishpingo » fait partie du stock de termes relatifs à la moitié roa adifo. Il permet donc au chanteur de se référer à son frère, roa adifo comme lui-même, sans prononcer son nom propre. II est clair néanmoins que la valeur de cette substitution ne se limite pas à un évitement négatif mais qu'elle est également dotée d'un aspect positif : le terme « ishpingo » véhicule des connotations de résistance et de virilité qui, sans être explicitées par l'exégèse du chanteur, ne sont pas moins présents.

Nous avons vu, dans les premières lignes de ce texte, que Percy était allé rendre visite à son frère qui vivait au Brésil, à l'aval du fleuve Purus — c'est précisément cet évènement qui est ici raconté. Ainsi, le contexte spatial du discours rapporté apparaît à plusieurs reprises : « au loin » $(10-11,33,40)$, « en aval de ce fleuve » (2-3, 69-70). Le déictique da précise qu'il s'agit de «ce fleuve », le Purus, à portée de regard lors de l'énonciation, présente et passée, du chant. Et l'objet du voyage est spécifié une fois : Percy pensait alors qu'il lui serait possible de vivre avec son frère (56-59).

Ce territoire lointain, situé en aval du fleuve Purus, est peuplé de métis (caboclos) brésiliens ; c'est d'eux dont il est question lorsque l'expression " étrangers morts » (yaba nahua, 5-6, $12,19,28,34,47,64,72,76)$ est employée. II s'agit là d'un substitut commun à tous les chants de ce genre : sa motivation est oubliée - les Sharanahua ne savent guère expliquer pourquoi ces étrangers sont associés de manière systématique aux morts.

Cependant, encore une fois, l'usage d'une telle expression n'est pas innocent : ces morts ne peuvent être que des revenants, catégorie bien définie chez les Sharanahua, où ils sont des êtres caractéristiques par au moins deux traits spécifiques : ils sont difficiles à reconnaître ou du moins ils apparaissent dans des contextes où la perception est brouillée (c'est ainsi qu'il est possible d'interpréter les question rhétoriques du type «Qui sont-ils ? », 10-11, 33, 755 ) et ils sont effrayants et dangereux. Ces deux traits propres aux revenants ne manquent pas de désigner, dans le contexte des évènements rapportés dans le chant, les étrangers 6.

Le cryptage du langage se concentre donc sur les personnes auxquelles se réfère le chant : le frère du locuteur, qui apparaît comme un auditeur, et les métis brésiliens, qui sont l'objet des paroles rapportées. Par la suite, le reste du chant décrit, dans une langue plutôt claire, les relations qui lièrent le locuteur aux étrangers : il souhaitait les découvrir (« je voudrais les voir », 13-14, 35-36, 77), il a tenté d'adopter leur mode de vie (« les lointains étrangers, je suis devenu [comme eux]», 28-31, 47-49, 72-73) mais ils ne l'ont pas reconnu comme I'un des leurs (« ils m'ont rejeté », 21-23, 64-66)7.

5 Un procédé similaire est employé au sein des chants initiatiques lorsqu'il est question des « esprits maîtres » (ifo) qui sont nommés yaba (Déléage, 2005, tome $1: 220-222$ ).

6 Il faudrait aussi préciser que certains Sharanahua ont l'habitude de dire que les « âmes des morts » (furoyoshi) s'en vont au Ciel en se dirigeant d'abord vers l'aval du fleuve, ce qui renforce le lien entre étrangers et morts.

7 Il faut préciser que les chanteurs sharanahua font une distinction entre l'ordre des lignes au sein d'un seul bloc de paroles citées (nous avons fait ressortir ces blocs en sautant une ligne entre chacun d'eux) et l'ordre de ces blocs entre eux. Seul le premier ordre exprime une logique narrative et temporelle ; le second ordre peut être présenté relativement arbitrairement (ainsi, les répétitions ne semblent pas signifiantes). 


\section{SECOND CHANT, PAR PERCY, DE LA MOITIÉ ROA ADIFO}

1 shaduhuahuun shadufo shaduhuahuun shadufo shaduhuahuun shadufo shaduhuahuun shadufo

5 shaduhuahuun yoidi yoidira yama yama yama da ua chacashta uhuun shaducairi biaquian yoipai yoipaira yama

10 yama yama

« uhuun shaducairi uhuun shaducairi mana futsa caifo uqui acutiashon

15 uqui acutiashon uhuun shaducairi ohuatisho duhuacai » huahuacaitiroba tirobara yama yama yama

20 « da yora osho budo yora osho uhuun shaducairi uhuun shaducairi ohuatisho duhuacai »

25 huahuacaidibahuai yama yama «shaduhuahuun shadufo shaduhuahuun shadufo da yora osho

30 da yora osho budo yora osho dicashofo shadufo dicashofo shadufo » shaduhuahuun huahuadi huahuadira yama

35 yama yama

« tsoa ishta yoiquin a yoidicohuin les petits oiseaux, les petits oiseaux les petits oiseaux, les petits oiseaux les petits oiseaux, les petits oiseaux les petits oiseaux, les petits oiseaux petits oiseaux, j'ai parlé, parlé yama yama yama

je suis triste mon petit oiseau j'ai souhaité te parler, te parler yama yama yama

« mon petit oiseau mon petit oiseau une autre colline bien qu'elle vive avec moi bien qu'elle vive avec moi mon petit oiseau je ne peux l'abandonner » ainsi ai-je pu, ai-je pu dire yama yama yama

« approche ce corps approche ce corps abîmé mon petit oiseau mon petit oiseau je ne peux l'abandonner » ainsi ai-je dû dire yama yama

« les petits oiseaux, les petits oiseaux les petits oiseaux, les petits oiseaux approche ce corps approche ce corps approche ce corps abîmé écoutez-moi, petits oiseaux écoutez-moi, petits oiseaux » petits oiseaux, ainsi ai-je dit, ainsi ai-je dit yama yama yama

« je ne le dis à personne je l'ai dit 
a yoidicohuin

adicofibacai

40 uhuun shaducairi

uhuun shaducairi »

shaduhuahuu yoipai

shaduhuahuu yoipai

shaduhuahuu yoipai

45 yama yama

«mana futsacaifo

mana futsacaifo

uqui acutiashon

uqui acutiashon

50 uhuun shaducairi

ohuatisho duhuacai »

huahuacaitiroba

huahuacaitiroba

yama yama

55 isca ishta aido

isca ishta aido

shaduhuahuun yoidi

shaduhuahuun yoidi yoidira yama

yama yama

60 « da yora osho

da yora osho

dicashohuu shadufo

dicashohuu shadufo »

shaduhuahuun huahuadi

huahuadira yama

65 yama yama

isca ishta aido

uhuun shaducaira

uhuun shaducaira

munquian yoidi yoidira yama

70 yama yama

« ano ocu rahuucai

ano ocu rahuucai

noco shadu inipa

noco shadu inipa

75 no iqui nonaimu»

shaduhuahuun huahuadi

huahuadira yama je l'ai dit

ils ne m'écoutent pas

mon petit oiseau

mon petit oiseau »

petits oiseaux, j'ai souhaité parler

petits oiseaux, j'ai souhaité parler

petits oiseaux, j'ai souhaité parler

yama yama

« une autre colline

une autre colline

bien qu'elle vive avec moi

bien qu'elle vive avec moi

mon petit oiseau

je ne peux l'abandonner »

ainsi ai-je pu dire

ainsi ai-je pu dire

yama yama

ainsi

ainsi

petits oiseaux, j'ai parlé

petits oiseaux, j'ai parlé, j'ai parlé yama

yama yama

« approche ce corps

approche ce corps

écoutez-moi, petits oiseaux

écoutez-moi, petits oiseaux »

petits oiseaux, ainsi ai-je dit, ainsi

ai-je dit yama

yama yama

ainsi

mon petit oiseau

mon petit oiseau

j'ai parlé ainsi, parlé ainsi yama

yama yama

« au loin, ensemble

au loin, ensemble

notre parfum de petits oiseaux

notre parfum de petits oiseaux

nous nous humons »

petits oiseaux, ainsi ai-je dit, ainsi ai-je dit yama 


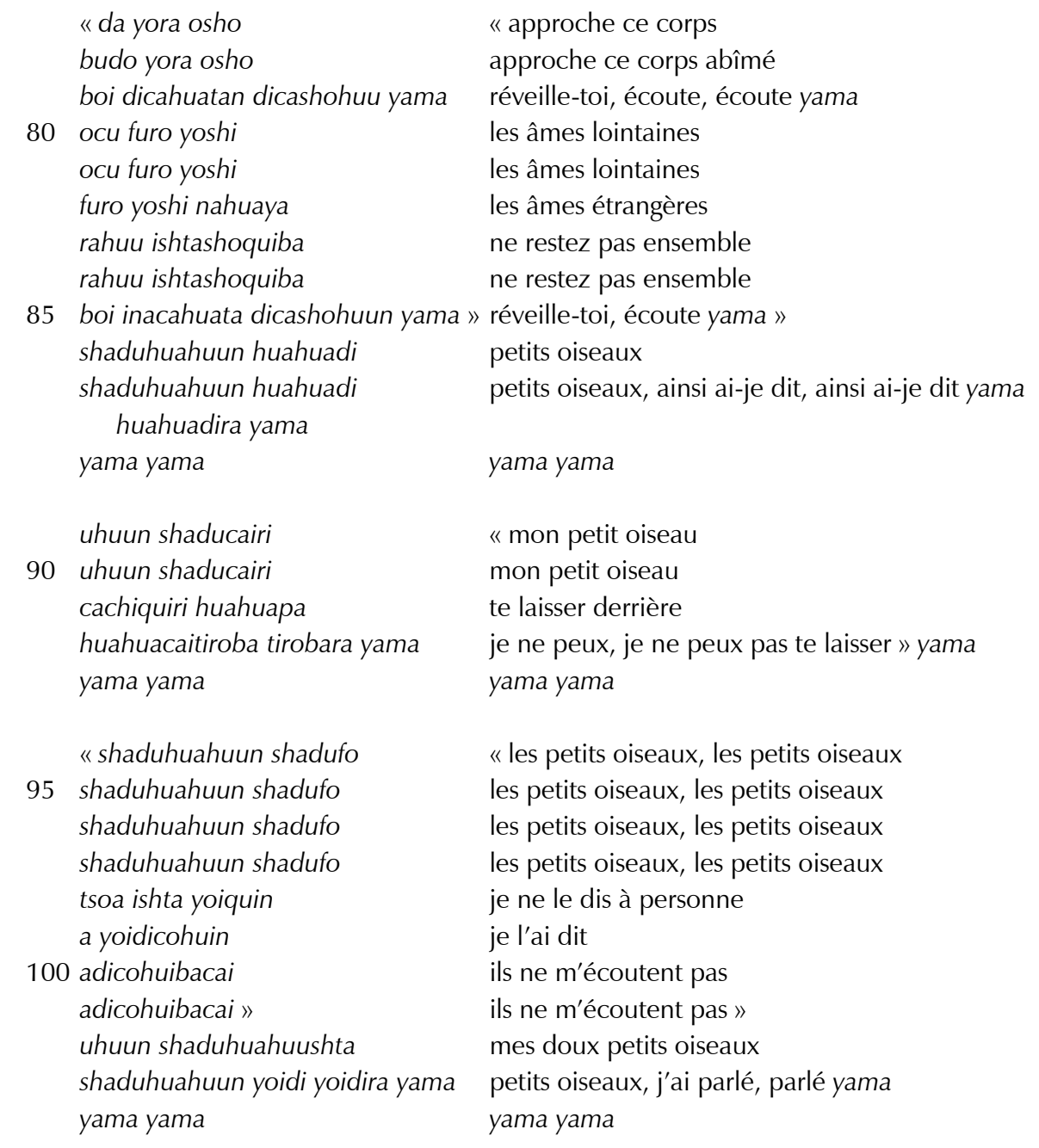

\section{APERÇU GLOBAL ET DÉCRYPTAGE}

Comme dans le premier chant, nous avons affaire à un texte presque entièrement composé d'auto-citations. Les conditions d'énonciations sont ainsi exprimées de manière identique, même si la forme diffère. Ces énoncés, équivalents au « Ainsi ai-je dit » du premier chant, sont : « Ainsi ai-je pu dire » $(18,52-53)$, « Ainsi ai-je dû dire » (25), « Ainsi ai-je dit » (34, $64,76,87)$, « J'ai parlé ainsi » $(5,57-58,79,104)$ et « J'ai souhaité te parler » $(9,42-44)$. ॥ s'agit donc, encore une fois, de rapporter les paroles passées du chanteur, prononcées au cours d'une période de grande tristesse (7).

L'épisode qui forme le sujet de ce chant appartient néanmoins à un genre distinct du voyage : il s'agit ici de la vie amoureuse du chanteur. Les discours rapportés furent adressés d'une part à la famille de l'épouse du chanteur et d'autre part à son épouse elle-même. Ainsi, quand le chant invoque, de manière litanique, des «petits oiseaux » (shadufo ou 
shaduhuahuun, 1-5, 27-35, 42-44, 57-58, 60-64, 86-87, 94-104), I'expression doit être comprise comme le terme « ishpingo » du premier chant : il s'agit d'un substitut permettant de faire référence à des membres de la moitié exogamique nahua facufo. Dans ce contexte, les «petits oiseaux » sont les membres féminins de la famille de l'épouse (nous verrons qu'il s'agit en fait de ses deux sœurs) ; ils font partie des destinataires passés du chants ( Écoutez-moi, petits oiseaux », 32-33). Toutefois, lorsque le terme est utilisé au singulier, shadu, il ne se réfère qu'à l'épouse du chanteur $(8,11-19,20-26,36-41,46-51,66-70$, 89-93) qui est la destinataire de toutes les paroles rapportées.

De quoi parlent ces discours ? De la réconciliation qui unit à nouveau le chanteur et son épouse, suite à une aventure extraconjugale. Pendant un temps, Percy vécut avec une autre femme, délaissant momentanément son épouse. Il pensa même la quitter définitivement; mais une conjonction compliquée de facteurs divers le poussèrent à regagner son foyer habituel. II lui fallut alors se réconcilier non seulement avec sa femme mais aussi avec sa famille.

Cette infidélité n'est mentionnée que deux fois, dont une au commencement du chant, et ce de manière très allusive : " Une autre colline, bien qu'elle vive avec moi, mon petit oiseau, je ne peux I'abandonner » (13-17, 46-51). Il faut comprendre que Percy vivait alors avec une autre femme ( une autre colline ») mais qu'il décida de ne pas abandonner sa légitime épouse (« mon petit oiseau »). Le terme " colline» (mana) désigne, de manière apparemment arbitraire, les femmes en général, quelle que soit leur moitié exogamique. En I'utilisant, Percy se met dans la situation de ne pas avoir à donner trop de détails sur I'identité de cette autre femme.

Puisqu'il «ne peut abandonner » sa femme, le chanteur retourne vers elle et lui demande humblement de l'accepter à nouveau comme époux : " Approche ce corps abîmé, petit oiseau »(20-21, 29-31, 60-61, 77-78). C'est bien sûr l'énonciateur que désigne, par métonymie, l'expression " corps abîmé » (budo yora) - ce qui n'est qu'une figure de style destinée à manifester une certaine humilité. La manœuvre aboutit : Percy parvint à se réconcilier avec son épouse qui l'accueillit à nouveau dans son hamac: " Au loin, ensemble, notre parfum de petits oiseaux, nous nous humons » (71-75). L'énoncé doit être compris comme la description des retrouvailles corporelles du couple : c'était il y a longtemps déjà (« au loin ») et Percy pu à nouveau apprécier le parfum caractéristique de son épouse.

Deux éléments du chant restent à clarifier. Le premier consiste en la promesse que fit Percy à sa femme et à sa belle famille de ne plus parler de cette histoire : c'était une des conditions de la réconciliation et une manière d'effacer le passé (36-41, 94-101). Ces évènements ne pourraient dès lors plus être évoqués que de manière détournée, par exemple par le biais des *yama yama.

Le second élément fait intervenir encore plus spécifiquement la famille de l'épouse : Percy pria alors sa femme de ne plus écouter la famille qui lui conseillait, réellement ou de manière rhétorique, de refuser son retour. Nous ne sommes pas parvenus à éclaircir définitivement ce point, mais il semble qu'à cette époque le père et la mère de l'épouse étaient déjà morts. Ce qui signifierait d'abord qu'il faudrait exclure la mère des « petits oiseaux » avec lesquelles eurent lieu les discussions (ce qui est logique dans la mesure où elle appartenait à la moitié roa adifo) et ensuite qu'une partie des tractations reposait sur un appel à l'autorité des parents décédés. On peut alors identifier les « petits oiseaux » aux deux sœurs de l'épouse qui, à cette époque, vivaient dans le même village. Une telle interprétation, qui nous fut suggérée par une personne connaissant peu les détails de la vie du chanteur mais très bien la technique des *yama yama, permettrait de comprendre les lignes suivantes : 
« Les âmes lointaines, les âmes étrangères, ne restez pas ensemble, réveille-toi, écoute » (80-85).

Les rencontres avec les morts se déroulent de manière privilégiée au cours des rêves ; l'énoncé pourrait donc être glosé ainsi :

« N'écoute pas tes défunts parents; sors du sommeil au cours duquel ils te parlent ».

Ce faisant, Percy détourna son épouse de l'idéal éthique de ses parents et fit en sorte que celle-ci ainsi que ses soeurs acceptent de l'écouter.

\section{DISCUSSION}

Deux principaux aspects sont susceptibles de susciter l'étonnement de l'auditeur ou du lecteur de cette tradition d'autobiographie chantée : d'une part le contenu de la narration autobiographique se réduit à quelques éléments assez pauvres au détriment de toute la richesse des évènements auxquels elle se rapporte, et d'autre part la formalisation extrême des énoncés rend difficilement crédible une interprétation littérale des conditions d'énonciation telles que présentées dans le chant lui-même.

\section{1. Relations autobiographiques}

En effet, les chants *yama yama sont avares de détails : ils se concentrent sur un petit nombre de termes et de relations. Le premier chant introduit le frère du chanteur (« ishpingo ») et les étrangers (« étrangers morts ») ; le second l'épouse du chanteur (« petit oiseau »), sa famille (« petits oiseaux » et « âmes étrangères ») et, rapidement, l'amante (« colline »). Ce sont ces termes, à l'exclusion de tout autre, qui sont codés de manière cryptique.

Les relations qu'entretient le chanteur avec ces termes ou ces personnes sont elles-mêmes fort limitées. Les premières sont de l'ordre de l'énonciation : le locuteur s'adresse à tel ou tel auditeur ; les secondes, qui forment l'objet des énoncés rapportés, sont également très limitées en nombre : « vouloir voir », « devenir », « être rejeté », et « vivre avec », « ne pas pouvoir abandonner », « approcher », « écouter », « humer», « ne dire à personne ». ॥ est donc légitime d'affirmer que ces chants ne portent que sur les relations qu'entretint le locuteur avec les différentes personnes qu'il invoque, ce qui appauvrit considérablement le contenu informatif de ces autobiographies.

Une étude plus attentive du texte des chants permet de comprendre ce qui est en jeu dans l'énoncé de ces relations. Dans le premier chant, il apparaît que la relation du chanteur aux étrangers n'est qu'un moyen d'exprimer les difficultés de la relation qu'il essaya d'établir avec son frère. Car il est clair que ce à quoi il a échoué (adopter le mode des vie des étrangers et s'intégrer parmi eux) a été réussi par son frère. Ce faisant, Percy l'identifie aux étrangers, et les relations du chanteur aux métis lui permettent d'exprimer, de manière indirecte et polie, les relations qu'il entretint avec son frère. La même séquence de trois relations est ainsi aisément transposable : Percy souhaitait revoir son frère et devenir comme lui, mais ce dernier l'a rejeté (pour des raisons qui nous sont restées obscures). L'apparente simplicité du chant recouvre donc un certain degré de complexité relationnelle fondé sur une identification implicite.

De ce point de vue, le second chant exprime des relations aussi complexes avec une grande économie de moyens. La relation du chanteur à son amante passée est à peine évoquée : 
les paroles du chant se concentrent essentiellement sur les relations unissant le chanteur, son épouse et la famille de cette dernière. Il y est donc moins question d'une aventure extraconjugale que de la réconciliation qui lui fit suite. Les énoncés tendent à identifier l'épouse (" mon petit oiseau ») à ses sœurs (« les petits oiseaux ») car il est question des mêmes relations : elles sont d'une part des auditrices et d'autre part l'objet de la tentative de rapprochement du chanteur (comparer 20-25 et 27-33) — seul le rapprochement corporel («nous nous humons », 71-75) doit être exclu de cette identification de l'épouse à ses sœurs. Par contre, leurs défunts parents sont délibérément situés en dehors de cette identification relationnelle : I'injonction «ne restez pas ensemble» (83-84) portant sur la relation entre l'épouse et ses parents entre en contradiction avec le résultat de la réconciliation entre elle et Percy, « au loin, ensemble» (71-72).

\section{2. Citation et apprentissage}

Cette analyse du contenu informatif des chants *yama yama en termes de simples relations susceptibles de se superposer les unes aux autres, dans un langage somme toute réduit ne manque pas de poser le problème du statut énonciatif des paroles citées. Nous avons vu que la quasi-totalité des ces chants se présentait comme des citations des paroles passées du chanteur. Pourtant, il apparaît peu vraisemblable que Percy ait un jour prononcé ces paroles, dans la forme au sein de laquelle elles sont rapportées. Non seulement les interactions qui furent réellement vécues semblent être présentées ici simplifiées à l'extrême, sous la forme de relations génériques, mais de plus, l'utilisation d'un langage fortement formalisé pour répéter ces paroles rend problématique le sens qu'il faut conférer aux énoncés du type «Ainsi ai-je dit».

Car le vocabulaire et la structure des chants *yama yama font l'objet d'un véritable apprentissage reconnu comme tel. Nous n'avons pas pu observer de telles situations $d^{\prime}$ enseignement dans la mesure où ce genre de tradition n'intéresse plus guère les jeunes générations. Nous en avons néanmoins obtenu quelques descriptions de la bouche de plusieurs hommes âgés, dont Percy lui-même. Dans tous les cas, le récit était le même : un jour, le père du locuteur avait décidé d'enseigner la technique des chants autobiographiques à son enfant. Ils s'étaient alors isolés dans un endroit tranquille de la maisonnée. Le père avait introduit ses chants par la formule suivante : "Un jour, tu chanteras ainsi ; c'est ainsi que me l'a enseigné mon propre père ». Suivait alors le chant lui-même que l'enfant devait répéter afin de l'apprendre progressivement par cœur. Lorsque ce dernier butait sur l'opacité de certaines expressions, son père les lui expliquait : «Ceci signifie cela et cela signifie ceci ». De cette manière, le jeune apprenti comprenait le sens des «métaphores » qu'il allait utiliser dans ses propres chants et il devenait capable de décoder celui des autres. C'est ainsi que Percy avait appris les chants de son père.

Tous nos interlocuteurs insistèrent sur un point étonnant : cet apprentissage était pensé comme une stricte imitation. Selon leurs paroles, il ne s'agissait pas d'improviser un chant à chaque fois nouveau à partir de la transmission d'un schéma narratif et d'une série de substituts codés. Tous affirmèrent que le but de l'enseignement était d'apprendre à répéter les chants verbatim et que c'est précisément la correction de cette répétition qui non seulement validait le chant en tant qu'appartenant à la tradition des *yama yama mais qui permettait aussi d'en évaluer la qualité esthétique.

Il est certes nécessaire de distinguer nettement la réalité de cette répétition à l'identique et les représentations qu'en entretiennent les chanteurs. L'écoute ou la lecture des textes font peser de sérieux doutes sur la plausibilité d'une répétition verbatim - même si la chose n'est pas impossible en soi. Cette représentation est toutefois fondamentale : elle constitue l'épistémologie explicite que les Sharanahua confèrent aux chants *yama yama. 
Le dispositif énonciatif de ces chants devient alors singulièrement complexe : d'une part les chants se présentent comme des citations des paroles passées du chanteur lui-même et d'autre part ils sont pensés comme des citations des chants traditionnels enseignés par les parents. À qui attribuer ces paroles ? Au père du chanteur ou au chanteur lui-même lorsqu'il était jeune homme ? Il nous semble que cette ambiguïté énonciative est un trait constitutif de la tradition sharanahua des autobiographies chantées. Elle permet de comprendre d'un côté pourquoi l'apprentissage d'un langage crypté est jugé nécessaire pour mettre en forme certains contenus autobiographiques et d'un autre côté quel est l'effet communicatif de ces chants.

\section{3. Langage crypté}

Car en effet, nous n'avons toujours pas compris pourquoi les Sharanahua jugèrent nécessaire d'élaborer une tradition de discours autobiographiques dans un langage largement opaque. Nous avons certes entrevu en partie les rôles qu'il jouait dans la structuration des *yama yama. Le premier a trait à la définition des personnes évoquées : I'utilisation de ces substituts dont la logique ne semble reposer que sur le genre et la moitié d'appartenance situe d'emblée l'évènement rapporté dans un champ relationnel prédéfini. Le second est en quelque sorte une valeur ajoutée : chacun des substituts, même lorsque sa motivation reste inconnue ou oubliée, véhicule des connotations qui stipulent des valeurs et des émotions stéréotypées, constituant une toile de fond aux évènements relationnels rapportés par le chant. Le frère-ishpingo évoque la virilité et une certaine rudesse dans les rapports émotionnels ; les étrangers-morts l'appréhension, la peur et les difficultés de recognition ; l'épouse-petit oiseau la vulnérabilité, la légèreté, mais aussi l'interminable bavardage. L'extension du domaine de ces connotations reste cependant implicite et elle est à la seule charge du locuteur ou de l'auditeur.

Ces deux caractéristiques des substituts trouvent leur plein développement dans l'exposé de séquences relationnelles qui sert de trame narrative aux chants *yama yama. Dans la mesure où le simple usage de ces termes établit le champ des relations possibles et les émotions et les valeurs liées à ces relations, on peut dire que le complexe de relations et d'émotions exposé dans les chants y est encapsulé. Les chants que nous avons présentés sont fondés sur des relations horizontales de sexes opposés : soit le frère, soit l'épouse du chanteur. Or ce qui motive le chant, c'est la rupture de la charge affective standard attribuée à ces relations. Ainsi, le lien unissant deux frères est, chez les Sharanahua, un lien d'entraide inconditionnel qui ici a été rompu : c'est le rejet de Percy par son propre frère, violation indéniable de l'ethos sharanahua, qui a engendré de fortes émotions et qui a rendu cet évènement mémorable — donc susceptible d'être reformulé au sein d'un *yama yama. De même, la relation entre un homme et son épouse doit normalement reposer sur la fidélité, même si celle-ci n'est qu'une façade. C'est donc à nouveau la violation d'une conduite normalisée qui a déclenché à la fois de puissantes émotions et leur remémoration. Dans les deux cas, on voit bien que seules certaines combinaisons relationnelles peuvent faire l'objet d'un souvenir formulable dans un chant *yama yama, par le biais d'un vocabulaire crypté et de formules relativement stéréotypées appris et adaptables à des contextes limités même s'ils sont à chaque fois différents.

Le langage crypté joue donc un rôle fondamental dans la mise en forme et dans la remémoration des *yama yama. II permet également de procurer un contexte stéréotypé aux relations et aux émotions qui font l'objet du chant. Or c'est l'usage de ce langage crypté qui rend légitime l'attribution des paroles des chants à la personne qui les a enseignées - par exemple au père de Percy. C'est ainsi que l'on peut comprendre le premier aspect du dispositif énonciatif : le locuteur attribue son chant à celui qui le lui a appris dans la 
mesure où le discours qui est rapporté diffère de celui qui a été effectivement prononcé. II est clair que Percy ne s'est adressé ni à son frère ni à son épouse dans les termes codés employés par son chant *yama yama. Cette différence s'explique si l'on veut bien accepter l'attribution de ce langage à une tierce personne, l'enseignant.

\section{4. Effet communicatif}

Il reste alors un ultime problème : pourquoi utiliser un dispositif énonciatif ambigu pour « exprimer » ses souvenirs ? Pour le résoudre, il faut s'intéresser aux circonstances de l'énonciation de ces chants. Percy avait beau insister sur le fait que ses chants ne s'adressaient à personne en particulier, qu'ils ne servaient qu'à exprimer, pour lui-même, le souvenir d'anciennes émotions, il n'en reste pas moins que, durant l'après-midi au cours duquel furent réalisés les enregistrements, deux auditeurs étaient présents : son épouse et nous-mêmes. Même si l'intention communicative n'était pas reconnue comme telle, la simple prononciation de ces paroles ne pouvait manquer d'être dotée d'un effet communicationnel.

Il faut donc savoir que la relation de Percy avec son épouse s'était quelque peu refroidie au cours des semaines précédant cet après-midi. Elle lui reprochait ses absences répétées et trop longues : s'il était normal d'effectuer quelques expéditions en amont du fleuve au cours de la saison sèche pour pêcher et récolter des œufs de tortue, Percy les avait multipliées, délaissant trop longtemps sa famille qui ne pouvait alors compter que sur la générosité de certains parents pour agrémenter leur repas de viande de gibier. Dans cette situation où les frictions étaient assez fréquentes, il devenait clair que Percy rappelait ses efforts passés en vue de se réconcilier avec sa femme dans une optique axée sur le présent. Le chant apparaissait donc comme une manière détournée de demander une réconciliation ; le processus d'identification prenait maintenant pour objet le dispositif énonciatif inclus dans le chant : en évoquant un discours de réconciliation passé, Percy prononçait un discours de réconciliation présent. En s'adressant à la personne que fut son épouse dans le passé, il tentait en fait de faire passer un message à la femme qu'il a à côté de lui.

Une analyse similaire est valable pour le premier chant que nous avons présenté. Les circonstances pertinentes sont à chercher dans la conversation que nous menions avec Percy. II nous avait raconté, en particulier, qu'il y a longtemps il avait été invité par une étrangère à visiter un lointain pays (les États-Unis). II n'avait alors pas osé quitter son village, à la fois par crainte de l'inconnu et parce qu'il avait une famille à charge. II était toutefois disposé, maintenant, à partir loin de chez lui, par exemple au Brésil ou même dans le pays de l'ethnologue qu'il avait en face de lui. Implicitement, Percy nous demandait de l'inviter et de prendre en charge son voyage et nous avions répondu, tout aussi implicitement, en disant que notre budget était limité et ne permettait que des dépenses ponctuelles, essentiellement en relation avec la recherche que nous menions, même s'il était évident que nous avions certes plus d'argent que lui. Dès lors, le chant *yama yama évoquant son voyage au Brésil et la manière dont son frère et, surtout, les étrangers, l'avaient rejeté nous était indirectement adressé : c'était une manière de nous faire comprendre sa déception en nous identifiant, de manière ambivalente, à son frère et aux étrangers.

Les chants *yama yama remplissent ainsi une très réelle fonction de communication. Mais, et c'est là l'important, il constitue un moyen de communication indirecte : non seulement, le chanteur peut se permettre de ne pas prendre en charge son discours en l'attribuant à la tradition d'enseignement dont il provient (ce qui est donné à percevoir dans la forme 
même du langage des chants), mais il laisse également à l'auditeur la possibilité d'agir comme s'il n'avait pas compris le message communiqué. La nature ambigu du dispositif énonciatif des chants et leur langage crypté n'ont ainsi d'intérêt que dans la mesure où ils permettent d'établir une relation de communication qui n'est explicitement reconnue ni par le locuteur ni par l'auditeur. On comprend alors pourquoi un tel apprentissage, coûteux, peut apparaître nécessaire : il permet de communiquer des choses qui ne pourraient être communiquées autrement.

Car, et ce sera là notre conclusion, un tel dispositif communicationnel n'a de sens que si l'on prend en compte l'éthique de parole que respectent les Sharanahua. II existe certaines choses qu'il est malséant de communiquer directement, dans la crudité du langage ordinaire. Il est difficile de décrire explicitement le domaine de ces sujets, même si l'on en acquiert rapidement une intuition adéquate. On pourrait avancer, de manière hypothétique, qu'il s'agit de sujets considérés comme délicats, souvent des demandes qui mettraient l'interlocuteur dans la position, extrêmement impolie, d'avoir à refuser nettement. Ou alors des sujets imprégnés d'émotions fortes ou compliquées qui pourraient induire des réactions excessives qu'il vaut mieux éviter de provoquer. On comprendrait alors mieux pourquoi les anciens évoquent régulièrement l'usage passé des chants *yama yama dans des contextes de séduction : de telles situations étaient certainement saturées de vives émotions et le risque du refus devait alors être vécu sur le mode de l'appréhension. $C^{\prime}$ 'est en tout cas à partir d'une telle éthique de ce qui peut être dit ou non, directement ou indirectement, que se révèle toute la valeur de la tradition des autobiographies chantées chez les Sharanahua.

\section{Remerciements}

Je tiens à remercier François Berthomé, Julien Bonhomme ainsi que les deux évaluateurs anonymes pour leurs remarques d'une grande pertinence.

\section{Références citées}

DÉLÉAGE, P., 2005 - Le chamanisme sharanahua ; Paris : École des Hautes Études Sociales Thèse inédite.

SCOTT, M., 2004 - Vocabulario sharanahua-castellano, 164 p. ; Lima : Instituto Lingüistico de Verano. 